Proceedings of the Online Conference "Applications of Physics in Mechanical and Material Engineering"

\title{
Research on Yb:YAG Laser Beam Power Distribution Used in Hybrid Processes
}

\author{
M. Kubiak*, T. DOMAŃski, \\ W. Piekarska And Z. SATERnus \\ Częstochowa University of Technology, 42-201 Częstochowa, Poland \\ Doi: 10.12693/APhysPolA.139.598 \\ *e-mail: kubiak@imipkm.pcz.pl
}

\begin{abstract}
The aim of this work is to determine the influence of Yb:YAG laser beam power distribution on the formation of a fusion zone in a laser-arc hybrid welded plate made of S355 steel. The laser beam heat source is modeled based on the geostatistical kriging interpolation method. The electric arc is modeled using classic Goldak's heat source power distribution. The hybrid heat source energetic parameters are assumed with respect to experimental research on Yb:YAG laser beam characteristics as well as on electric arc current and voltage obtained during the real heating tests.
\end{abstract}

topics: hybrid heat source, Yb:YAG laser, beam caustics, electric arc

\section{Introduction}

Changes of focusing in the optical system of a laser beam change a laser spot diameter as well as a laser beam power intensity distribution. A part of the pumped laser energy converts to a heat that acts as the heat source inside a laser material. This heat source contributes to the non-uniform temperature distribution in the gain medium which is dependent on the gain medium configuration and cooling geometry [1]. This, in turn, contributes to the radial heat dissipation and induces no desirable thermal lensing effects influencing the beam shape, quality and output power stability [2]. The above and many other issues concerning the energetic characteristic of a laser beam are currently under particular investigation in the field of mathematical modeling and experimental research $[3,4]$.

A laser thermal load transferred through the material generates high temperature gradients and rapid cooling of steel which has the impact on the structural composition and mechanical properties of the processed material. Therefore, hybrid laser-arc processing is used to reduce drawbacks of a single laser beam acting on a material [5].

The aim of this work is the numerical analysis of the influence of Yb:YAG laser beam power distribution in the function of laser beam caustics on the temperature distribution in laser-arc hybrid heating of flat elements made of S355 steel.

\section{Experimental research}

The research on laser beam power distribution as well as on current and voltage of the electric arc in the hybrid laser-arc material processing is performed using a Trumpf D70 laser head combined with a TruDisk 12002 disk laser and a MIG torch. Energetic laser beam parameters are obtained using a Prometec UFF100 beam analyzer for measuring of a high power laser beam profile (Fig. 1).

The measurement is made for different beam focal points from $z=0$ (at the top surface of the heated element) up to $z=10 \mathrm{~mm}$, with the accuracy of $1 \mathrm{~mm}$. Distributions of the laser beam heat source for the chosen focusing are presented in Fig. 2.

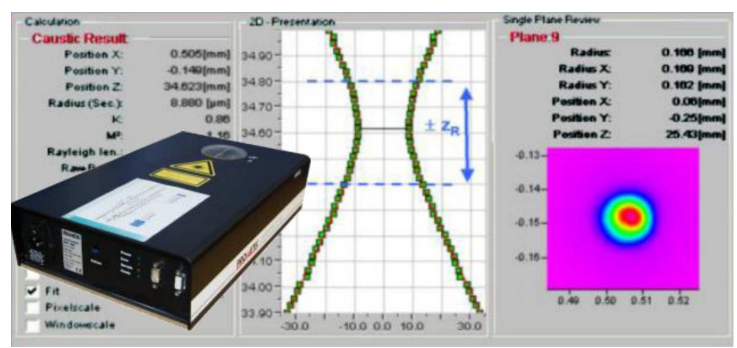

Fig. 1. Laser beam analyzer UFF100.
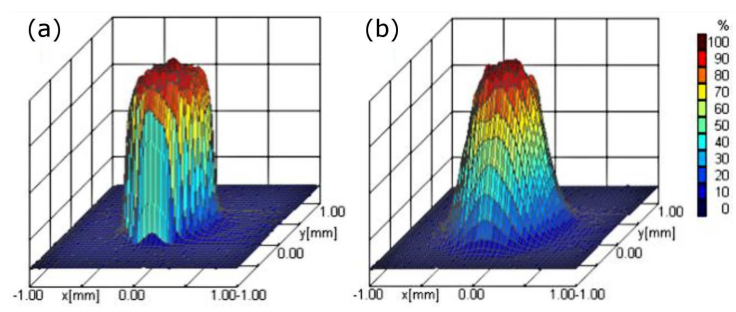

Fig. 2. Percentage laser beam power distribution for focusing (a) $z=0$ and (b) $z=5 \mathrm{~mm}$. 


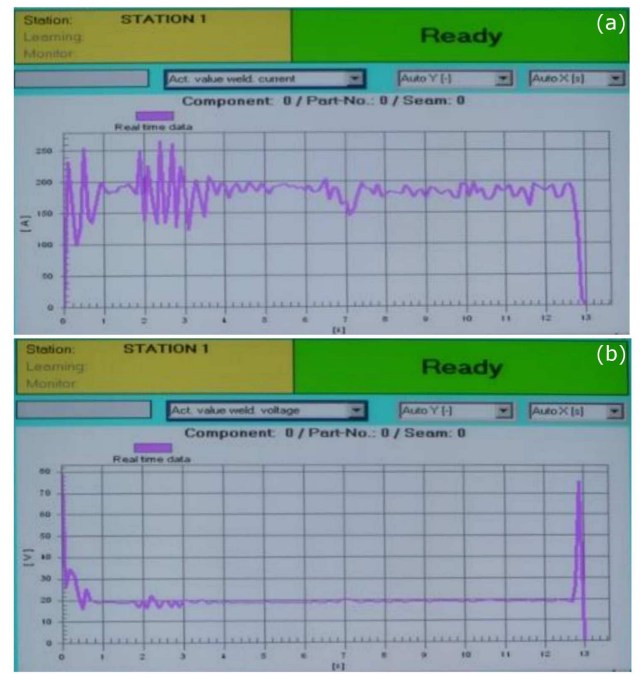

Fig. 3. Measured (a) current and (b) voltage of the electric arc.

Electric arc parameters (current and voltage) are measured during the experiment (Fig. 3). These parameters are further used in computer simulations.

\section{Heat source model}

The kriging interpolation method at the point $(x, y)$ is a linear combination of observations. The estimate is a function of the weighted average:

$$
\tilde{f}(x, y)=\sum_{i=1}^{n} w_{i} f\left(x_{i}, y_{i}\right)
$$

where $w_{i}$ are the weight coefficients assigned to particular observations, $f\left(x_{i}, y_{i}\right)$ is the real value of the function (variable) at the measured point and $n$ is the number of sampling points that are considered in estimating of the variable within the circle of radius $r_{k}$ from the estimated point.

The coefficients $w_{i}$ are calculated on the basis of the kriging system of equations [6], where the theoretical semivariogram is unknown and typically approximated by analytical functions. One of the main functions used in the approximation is a linear function $\gamma(h)=C_{0}+h S$ (Fig. 4), in which the semivariogram tends to sill $S$ at $h \rightarrow \infty$, while $C_{0}$ is a function discontinuity (nugget effect).

The double ellipsoidal power distribution of the electric arc heat source [7] is used in modeling of hybrid processes. This heat source is described by the following system of equations:

$$
Q_{1}= \begin{cases}q_{1}(x, y, z)=\frac{6 \sqrt{3}}{(\pi)^{3 / 2}} \frac{f_{1} Q_{\mathrm{A}}}{a b c_{1}} \exp \left(-\frac{3 x^{2}}{c_{1}^{2}}-\frac{3 y^{2}}{a^{2}}-\frac{3 z^{2}}{b^{2}}\right) & \text { for } x<x_{0} \\ q_{2}(x, y, z)=\frac{6 \sqrt{3}}{(\pi)^{3 / 2}} \frac{f_{2} Q_{\mathrm{A}}}{a b c_{2}} \exp \left(-\frac{3 x^{2}}{c_{2}^{2}}-\frac{3 y^{2}}{a^{2}}-\frac{3 z^{2}}{b^{2}}\right) & \text { for } x \geq x_{0}\end{cases}
$$

where $a, b, c_{1}$ and $c_{2}$ are the set of axes defining the front ellipsoid and the rear ellipsoid, $f_{1}$ and $f_{2}$ $\left(f_{1}+f_{2}=2\right)$ represent the distribution of the source energy at the front and rear section of the source. Thus, the resultant distribution of the source energy is the total sum described as $Q_{1}(x, y, z)=$ $q_{1}(x, y, z)+q_{2}(x, y, z)$. The parameter $Q_{\mathrm{A}}=\eta_{\mathrm{A}} I U$ reads as the arc heat source power, where $I$ is the current intensity, $U$ is the voltage and $\eta_{\mathrm{A}}$ is the efficiency of the electric arc.

Arc + laser hybrid heat source distribution (Fig. 5) is considered as a product of "double ellipsoidal" Goldak's model and Yb:YAG interpolation model $\left(Q_{1}+Q_{2}\right)$.

\section{Numerical modeling of temperature field}

The governing equation describing the temperature field in a laser heated material is numerically solved using the finite element method (FEM) in the Petrov-Galerkin formulation. The weak form of the heat transfer equation is expressed as follows:

$$
\begin{gathered}
\int_{\Omega^{\prime}} \mathrm{d} \Omega \phi(\nabla \cdot(\lambda \nabla T)+\tilde{Q})= \\
\int_{\Omega^{\prime}} \mathrm{d} \Omega C_{\text {ef }} \phi\left(\frac{\partial T}{\partial t}+\nabla T \cdot \boldsymbol{v}\right),
\end{gathered}
$$

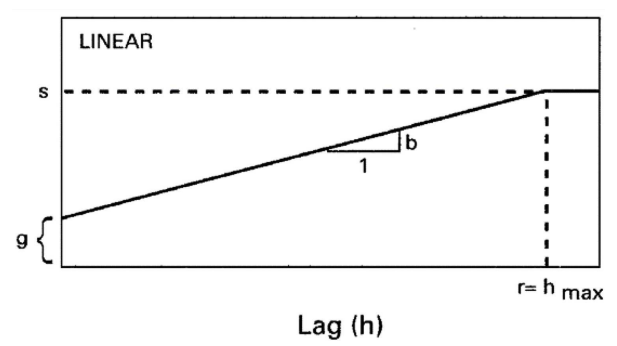

Fig. 4. Characteristic of the linear function used in the approximation of the theoretical semivariogram.

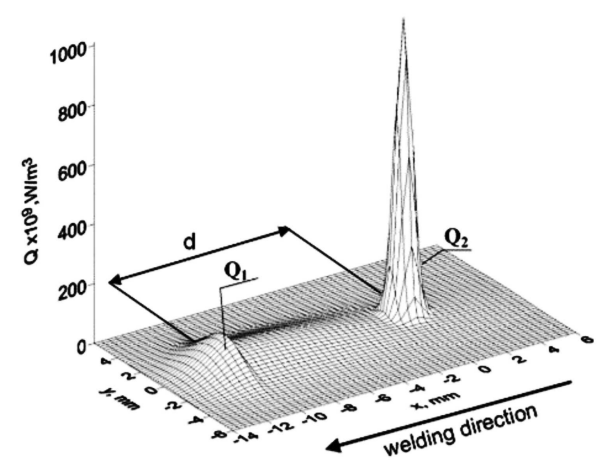

Fig. 5. Hybrid heat source power distribution at the top surface $(z=0)$ of the welded joint. 
where $T=T\left(x_{i}, t\right)$ is the temperature $[\mathrm{K}]$ at the point $x_{i}, \boldsymbol{v}$ is the velocity vector $[\mathrm{m} / \mathrm{s}], \lambda=\lambda(T)$ is the thermal conductivity, $C_{\text {ef }}=C_{\text {ef }}(T)$ is the effective heat capacity with the latent heat of fusion. In turn, the evaporation expressed as $\phi=\phi\left(x_{\alpha}\right)$ is the spatial weight function in the Petrov-Galerkin formulation.

Temperature in every node of finite element mesh is a time function $T_{j}=T_{j}(t)$. The integration of (3) over the time leads to the following form:

$$
\begin{aligned}
& \sum_{e} \int \mathrm{d} t \vartheta(t)\left[\left(K_{i j}^{e}+V_{i j}^{e}\right) T_{j}(t)+M_{i j}^{e} \partial_{t} T_{j}\right]= \\
& \sum_{e} S_{i j}^{e} \int \mathrm{d} t \vartheta(t) Q_{j}^{e}(t)-\sum_{e}^{\Gamma} S_{i j}^{\Gamma} \int_{t} \mathrm{~d} t \vartheta(t) \tilde{q}_{j}^{e}(t) .
\end{aligned}
$$

Here, $\vartheta(t)$ is the time weight function, $K_{i j}^{e}$ is the local thermal conductivity matrix, $V_{i j}^{e}$ is the local convection matrix, $M_{i j}^{e}$ is the local heat capacity matrix, $S_{i j}^{e}$ is the local coefficients matrix, $Q_{j}^{e}$ is the local vector of internal sources efficiency and $\tilde{q}_{j}^{e}$ is the local vector of boundary fluxes. Each term in (4) can be given separately, namely

$$
\begin{aligned}
& K_{i j}^{e}=\int_{\Omega^{\prime}}^{e} \mathrm{~d} \Omega^{e} \lambda^{e} \frac{\partial w_{i}}{\partial x_{\alpha}} \frac{\partial \phi_{j}}{\partial x_{\alpha}}, \\
& V_{i j}^{e}=C_{\mathrm{ef}}^{e} \int_{\Omega^{\prime}}^{e} \mathrm{~d} \Omega w_{i} \frac{\partial \phi_{j}}{\partial x_{\alpha}} v_{\alpha}, \\
& Q_{i}^{e}=S_{i j}^{e} Q_{j}=\int_{\Omega^{\prime}}^{e} \mathrm{~d} \Omega w_{i} \phi_{j} Q_{j}^{e}, \\
& M_{i j}^{e}=(\rho C)^{e} S_{i j}^{e}, \\
& \tilde{q}_{i}^{e}=S_{i j}^{\Gamma^{e}} \tilde{q}_{j}^{e}=\int_{\Gamma}^{e} \mathrm{~d} \Gamma^{{ }^{e}} w_{i}^{\Gamma^{\prime}} \phi_{j}^{\Gamma^{\prime}} \tilde{q}_{j}^{e}
\end{aligned}
$$

with the finite element number $e$, and $i$ and $j$ the nodes numbers.

\section{Results and discussion}

Computer simulation of a single laser beam and hybrid processing is performed for sheets made of S355 steel in the system with a leading laser beam heat source in the tandem. The dimensions of the

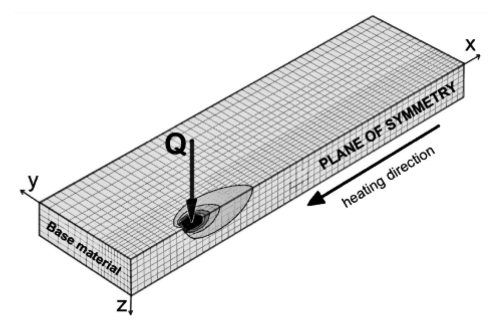

Fig. 6. Scheme of the considered system. sheets are $150 \times 40 \times 5 \mathrm{~mm}^{3}$. Simulations are performed with different laser beam focusing $z=0$ and $z=10 \mathrm{~mm}$. The scheme of the considered system is presented in Fig. 6.

\subsection{Interpolated heat source}

Results of a laser beam heat source interpolation for beam focusing $z=0$ are presented in Fig. 7 in the form of the comparison between the real measured beam distribution and the interpolated model. Figure 8 illustrates the interpolated laser beam heat source power distribution for beam focusing $z=10 \mathrm{~mm}$.

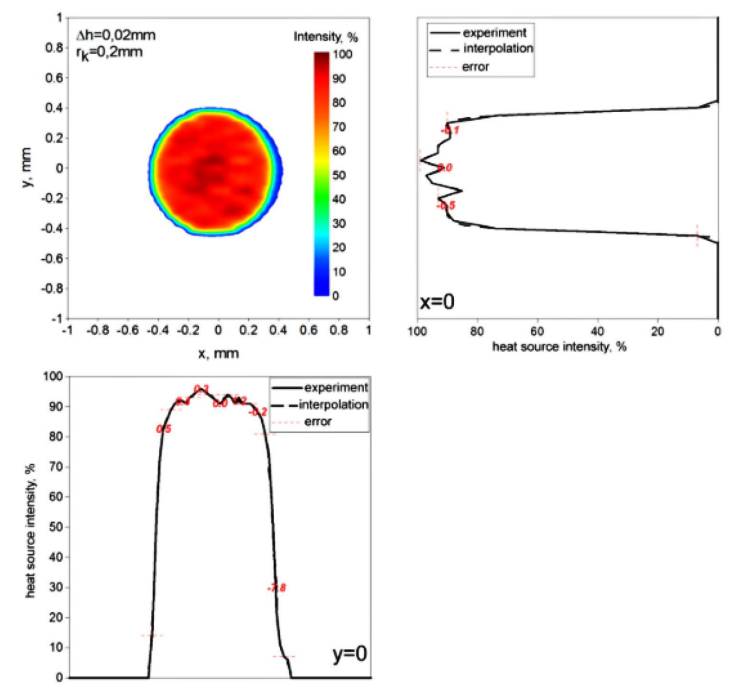

Fig. 7. Percentage distribution of laser power described by the interpolation model for $\Delta h=0.02 \mathrm{~mm}$. Beam focusing $z=0$.

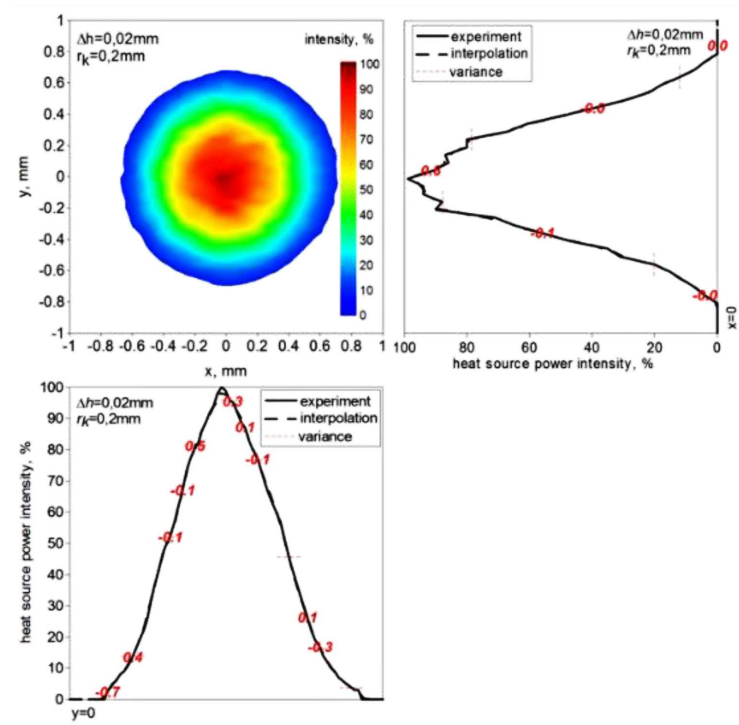

Fig. 8. Percentage distribution of laser power described by the interpolation model for $\Delta h=0.02 \mathrm{~mm}$. Beam focusing $z=10 \mathrm{~mm}$. 


\subsection{Single laser beam heating}

Temperature distributions in the longitudinal section of the laser heated element for $y=0$ (in the heating line) are presented in Fig. 9. Now, in Fig. 9a, the temperature field simulation results obtained using the analytical Gaussian laser beam power distribution are shown. In turn, the temperature field simulations obtained using the interpolated heat source model are shown in Fig. 9b. Each simulation is made for the same process parameters and beam focusing $z=0$.
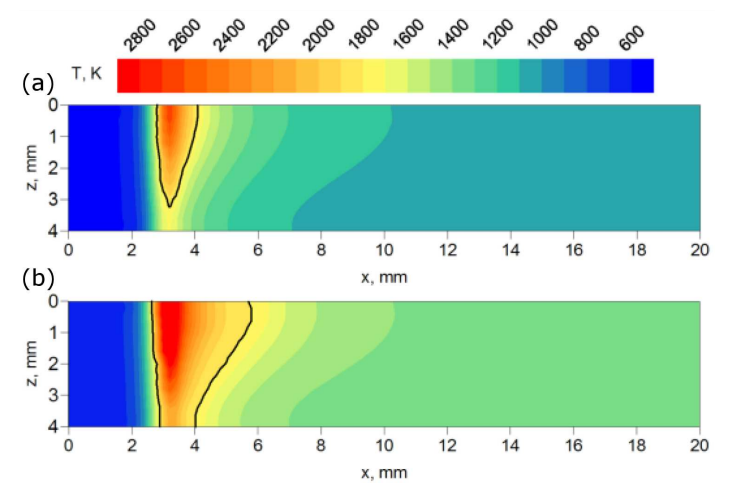

Fig. 9. Temperature distribution in the longitudinal section of the heated element $(y=0)$ for beam focusing $z=0$ using (a) analytical (Gaussian) heat source and (b) interpolated heat source.

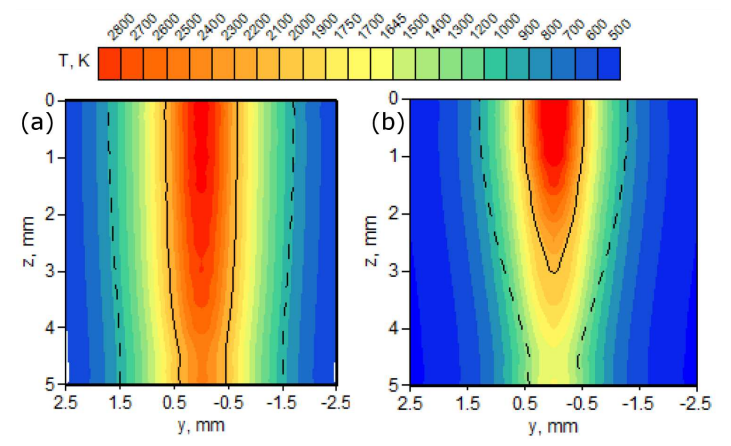

Fig. 10. Temperature distribution in the cross section of the heated element for beam focusing (a) $z=0$ and (b) $z=10 \mathrm{~mm}$.

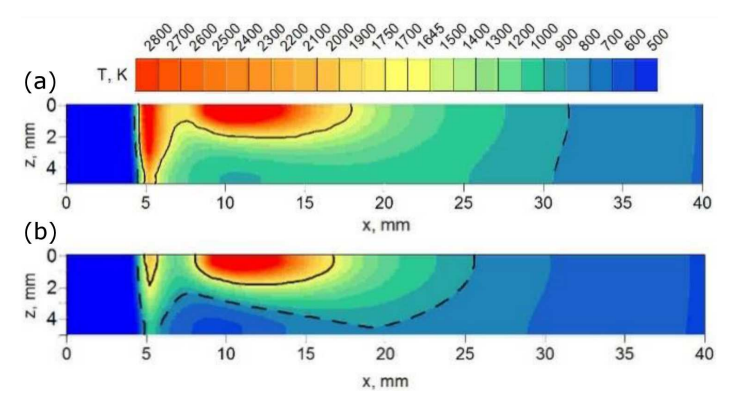

Fig. 11. Temperature distribution in the longitudinal section $(y=0)$ of the heated element for beam focusing (a) $z=0$ and (b) $z=10 \mathrm{~mm}$.
Figure 10 shows the temperature field in the cross section of the heated and melted element. Simulations are made for the interpolated heat source model and two different beam focusing: (a) $z=0$ and (b) $z=10 \mathrm{~mm}$.

In Figs. 9 and 10, the melted zone geometry is marked by a solid line, while the heat affected zone geometry — by a dashed line.

\subsection{Hybrid laser-arc beam heating}

Figure 11 presents temperature distributions in the longitudinal section of the hybrid laser-arc heated element for $y=0$ (in the heating line). In Fig. 9a, we show the simulated temperature field obtained for beam focusing $z=0$, and in Fig. 9b - the temperature field obtained for beam focusing $z=10 \mathrm{~mm}$.

\section{Conclusions}

From the data analysis in Fig. 9 it can be observed that the interpolated heat source model affects the temperature field and thus the predicted characteristic zones in the heated element (the melting zone and the heat affected zone). The comparisons in Figs. 10 and 11 show differences in the melting zone and the heat affected zone at different focusing of $\mathrm{Yb}$ :YAG laser beam. For beam focusing $z=0$, the synergy effect of heat sources is visible in Fig. 11. In the case of the defocused beam (Fig. 11b), there is no through material melting effect.

The significant impact of the heat source power distribution on the temperature distribution and melted pool as well as the heat affected zones is noticeable. This distribution depends on the focusing of Yb:YAG laser beam (other technological parameters are the same).

\section{References}

[1] J.C. Ion, Laser Processing of Engineering Materials, Elsevier ButterworthHeinemann, Amsterdam 2005.

[2] High Power Diode Lasers, Technology and Applications, Eds. F. Bachmann, P. Loosen, R. Poprawe, Springer Series in Optical Sciences, Springer, 2007, p. 128.

[3] M. Kubiak, W. Piekarska, Comp. Struct. 172, 29 (2016).

[4] M. Kubiak, W. Piekarska, S. Stano, Int. J. Heat Mass Trans. 83, 679 (2015).

[5] P. Seyffarth, I.V. Krivtsun, Laser-Arc Processes and their Applications in Welding and Material Treatment, Taylor \& Francis, USA 2002.

[6] M.A. Oliver, R. Webster, Int. J. Geogr. Inf. Syst. 4, 313 (1990).

[7] J.A. Goldak, Computational Welding Mechanics, Springer, New York, 2005. 